\title{
Transient solution of a thermoelastic instability problem using a reduced order model
}

\author{
Abdullah M. Al-Shabibi, J.R. Barber* \\ Department of Mechanical Engineering, University of Michigan, Ann Arbor, MI 48109-2125, USA
}

Received 24 April 2001; received in revised form 12 November 2001

\begin{abstract}
Above a certain critical speed, sliding systems with frictional heating such as brakes and clutches can exhibit thermoelastic instability in which non-uniform perturbations develop in the pressure and temperature fields. A method is described in which the transient thermomechanical behaviour of such systems is approximated by a reduced order model, describing one or more dominant perturbations or eigenfunctions. The goal is to construct a mathematical model of the system with a modest number of degrees of freedom.

If a single dominant perturbation is used, an integral expression can be written for the evolution of the perturbation with time. A more accurate description involving several terms requires that the transient behaviour be generated by a sequence of operations in which the sliding speed is piecewise constant. Both models are evaluated by comparison with a direct numerical simulation and prove to give good accuracy with a dramatic reduction in computing time. (C) 2002 Elsevier Science Ltd. All rights reserved.
\end{abstract}

Keywords: Brake; Clutch; Thermoelastic instability; TEI; Reduced order model; Eigenfunction series

\section{Introduction}

During brake application or engagement of a transmission clutch, frictional heat is generated and the resulting non-uniform temperatures cause thermoelastic distortion which in turn affects the contact pressure distribution. The system is unstable if the sliding speed is sufficiently high, in which case contact pressure perturbations grow, leading to localization of load and heat generation. This phenomenon is known as thermoelastic instability (TEI) [1]. Hot spots are then generated at the sliding interface, causing material damage, wear and low-frequency frictional vibrations [2-4].

\footnotetext{
${ }^{*}$ Corresponding author. Tel.: +1-734-936-0406; fax: +1-734-647-3170.

E-mail address: jbarber@umich.edu (J.R. Barber).
} 


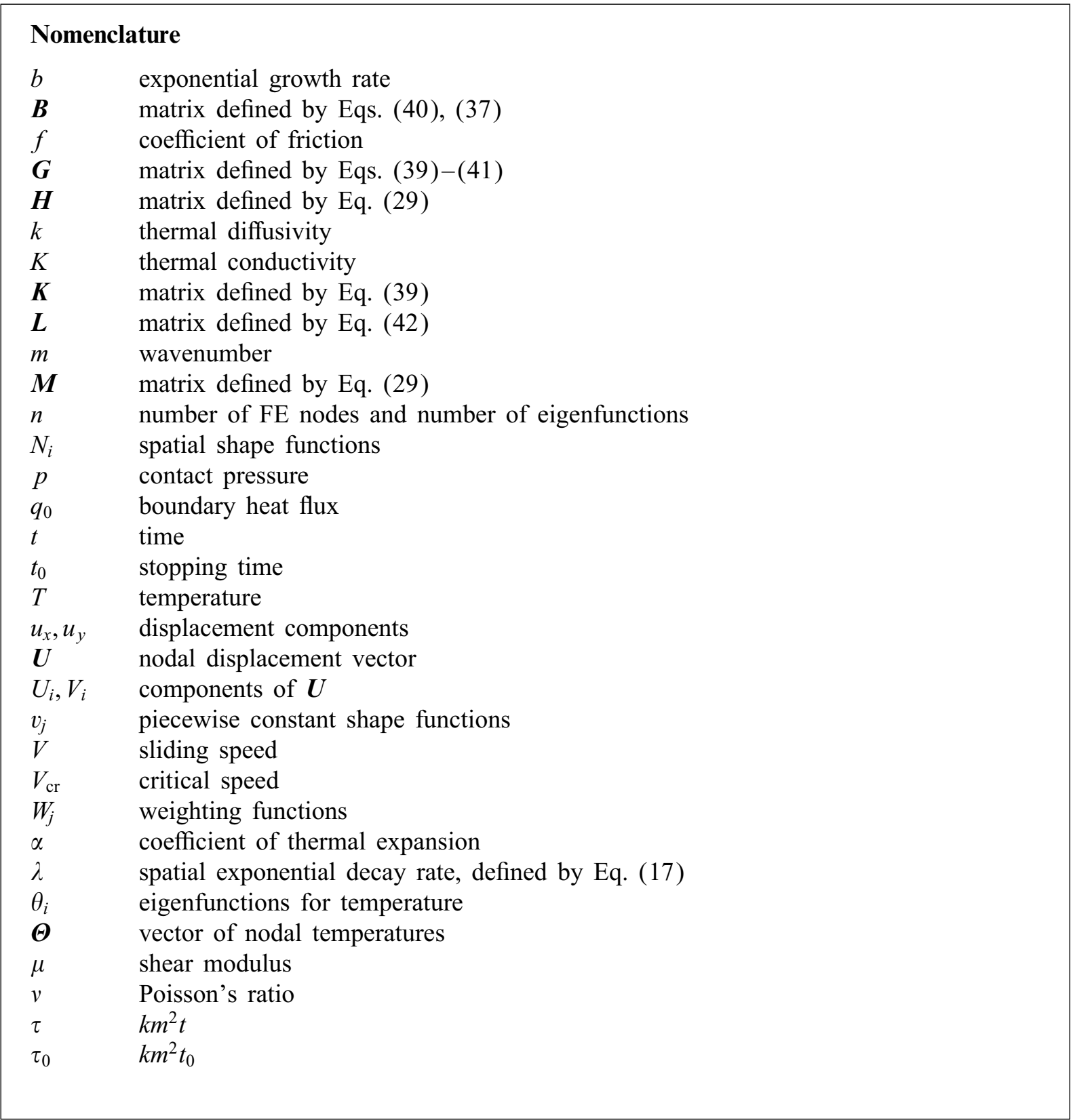

Methods for determining the critical sliding speed for instability were pioneered by Burton et al. [5] and have since been developed for practical brake and clutch geometries using the finite element method [6,7]. However, these solutions all assume that the sliding speed is constant, whereas actual brakes and clutches operate at variable sliding speed. The speed may be above critical at initial engagement, but dangerously high temperatures may not occur before the speed has reduced below 
critical [8]. Also, temperature perturbations may not have time to decay to a uniform state between engagements in complex clutch systems.

These effects might be simulated by finite element analysis [9,10], but transient simulations are very computer intensive. In the present paper, we explore some simpler approximate methods analogous to reduced order models used in the analysis of complex dynamic systems and based on the concept of a truncated eigenfunction expansion.

\section{The eigenfunction series}

Burton's method of investigating TEI is to identify solutions of the perturbation problem of the exponential form

$$
T(x, y, z, t)=\mathrm{e}^{b_{i} t} \theta_{i}(x, y, z),
$$

where $T$ is the temperature field in Cartesian coordinates $x, y, z$ and $t$ is time. Substitution of Eq. (1) into the equations of heat conduction and thermoelasticity and the boundary conditions leads to an eigenvalue problem for the exponential growth rate $b_{i}$ and the associated eigenfunction $\theta_{i}$.

A general solution for the transient evolution of a perturbation at constant sliding speed can be written as an eigenfunction series:

$$
T(x, y, z, t)=\sum_{i=1}^{n} C_{i} \mathrm{e}^{b_{i} t} \theta_{i}(x, y, z),
$$

where $n$ is the number of eigenvalues and $C_{i}$ is a set of arbitrary constants determined from the initial condition $T(x, y, z, 0)$. In a discrete (e.g. finite element) formulation, $n$ will be equal to the number of nodal temperatures, whereas for a continuum formulation it will be infinite. It follows from Eq. (2) that if at least one eigenvalue is positive or complex with positive real part, the perturbation will grow without bounds and the system is unstable in the linear régime.

Over time, the term with the largest value of $\mathfrak{R}\left(b_{i}\right)$ will dominate the transient response, so if the initial perturbation is sufficiently small, this is the only term which needs to be considered. Denoting its growth rate and eigenfunction as $b_{1}, \Theta_{1}$, respectively, we can then conclude that

$$
\frac{\partial T}{\partial t}=b_{1} \mathrm{e}^{b_{1} t} \theta_{1}=b_{1} T \text {. }
$$

These results apply if and only if contact is maintained at all times (without which the problem would become non-linear) and the sliding speed $V$ is constant. However, an approximate solution to the linear problem with variable speed can be defined by assuming that Eq. (3) still holds, where $b_{1}$ is now to be interpreted as the dominant eigenvalue at the instantaneous speed $V(t)$, denoted by $b_{1}(V(t))$. Solving the resulting differential equation, we then obtain

$$
T=T(0) \exp \left(\int_{0}^{t} b_{1}(V(t)) \mathrm{d} t\right),
$$

where $T(0)$ is the temperature at $t=0$. By a similar argument, the evolution of the perturbation in contact pressure can be approximated by

$$
p=p(0) \exp \left(\int_{0}^{t} b_{1}(V(t)) \mathrm{d} t\right),
$$

where $p(0)$ is the contact pressure at $t=0$. 


\subsection{Nature of the approximation}

Eqs. (4), (5) are only approximately true when the speed varies, because the form of the eigenfunction (e.g. the temperature distribution) also varies with speed. To understand the effect of this variation, consider a scenario in which the sliding speed $V$ is piecewise constant in time. In other words, the system experiences a sequence of time periods at different constant sliding speeds. In mathematical terms, we can write

$$
V(t)=\sum_{j=1}^{M} V_{j} v_{j}(t)
$$

where $v_{j}(t)$ are piecewise constant shape functions defined by

$$
\begin{aligned}
v_{j}(t) & =1, \quad t_{j-1}<t<t_{j} \\
& =0, \quad t<t_{j-1} \text { and } t>t_{j}
\end{aligned}
$$

and $t_{j}$ are a set of nodal times with $t_{0}=0$.

During the $j$ th time period, the sliding speed is constant and equal to $V_{j}$ and we can write the temperature field in the eigenfunction series

$$
T^{(j)}(x, y, z, t)=\sum_{i=1}^{n} C_{i}^{(j)} \mathrm{e}^{b_{i}^{(j)}\left(t-t_{j-1}\right)} \theta_{i}^{(j)}(x, y, z)
$$

where $b_{i}^{(j)}, \theta_{i}^{(j)}(x, y, z)$ are the eigenvalues and eigenfunctions, respectively, appropriate to speed $V_{j}$ and we have chosen to reset the zero for time in each time step.

Continuity of temperature at time $t_{j}$ then requires that

$$
T^{(j+1)}\left(x, y, z, t_{j}^{+}\right)=T^{(j)}\left(x, y, z, t_{j}^{-}\right)
$$

and hence

$$
\sum_{i=1}^{n} C_{i}^{(j+1)} \theta_{i}^{(j+1)}(x, y, z)=\sum_{i=1}^{n} C_{i}^{(j)} \mathrm{e}^{b_{i}^{(j)}\left(t_{j}-t_{j-1}\right)} \theta_{i}^{(j)}(x, y, z),
$$

from Eq. (9). In other words, at the end of each time step we need to re-expand the instantaneous temperature field as a series of the eigenfunctions appropriate to the next time step.

If the change in speed between time steps is relatively small, the new eigenfunctions will be quite similar to the old ones and the new dominant eigenfunction will still capture the greater part of the temperature distribution. However, some of the distribution will 'leak out' into decaying eigenfunctions. By contrast, Eq. (4) takes no account of the variation of the eigenfunctions with speed and might therefore be expected to overestimate the growth of the temperature.

The piecewise constant solution defined by Eqs. (9), (11) also represents a more accurate approximation to the transient solution, depending on the size of time step used. As such, it is an attractive alternative to numerical simulation, since the latter tends to be restricted by the necessity to use very small time steps to preserve numerical stability. This solution also opens up the possibility of defining an intermediate 'reduced order model' in which some but not all of the eigenfunctions in the series are retained, much in the same way as the response of a dynamic system can be approximated by a system capturing an appropriate selection of the natural modes of vibration. 
In the next section, we shall examine the viability of these ideas in the context of a simple example problem.

\section{Half-plane sliding against a rigid surface}

Fig. 1 shows the thermoelastic half-plane $y>0$ sliding against a rigid non-conducting body at speed $V(t)$, which may be a function of time. The two bodies are infinite in extent in the $x$-direction and are pressed together by a uniform pressure $p_{0}(t)$ applied at the extremities distant from the interface. Sliding friction occurs at the interface $y=0$ with coefficient $f$, leading to the generation of frictional heat

$$
q(x, t)=f V(t) p(x, t),
$$

where $p(x, t)$ is the contact pressure. Since the lower body is non-conducting, all of this heat must flow into the thermoelastic half-plane, resulting in the thermomechanically coupled boundary condition

$$
q_{y}(x, 0, t)=-K \frac{\partial T}{\partial y}(x, 0, t)=f V(t) p(x, t),
$$

where $K$ is the thermal conductivity of the half-plane.

This transient thermomechanical contact problem has a simple one-dimensional solution in which the contact pressure and the temperature field are independent of the $x$-coordinate. However, Burton et al. [5] have shown that if the speed is sufficiently high, this solution may be unstable, leading to the exponential growth of sinusoidal perturbations in temperature and pressure. For example, the contact pressure will then take the form

$$
p(x, t)=p_{0}(t)+p_{1} \mathrm{e}^{b t} \cos (m x) .
$$

Eventually, these perturbations will grow sufficiently large for separation to occur, after which the assumption of linearity will cease to apply.

\subsection{Growth of a sinusoidal perturbation}

Following Burton et al. [5], we consider cases in which the perturbation in the temperature field takes the sinusoidal form

$$
T(x, y, t)=\theta(y) \mathrm{e}^{b t} \cos (m x) .
$$

Substitution in the transient heat conduction equation yields the ordinary differential equation

$$
\frac{\mathrm{d}^{2} \theta}{\mathrm{d} y^{2}}-\lambda^{2} \theta=0
$$

for $\theta(y)$, where

$$
\lambda=\sqrt{m^{2}+\frac{b}{k}}
$$




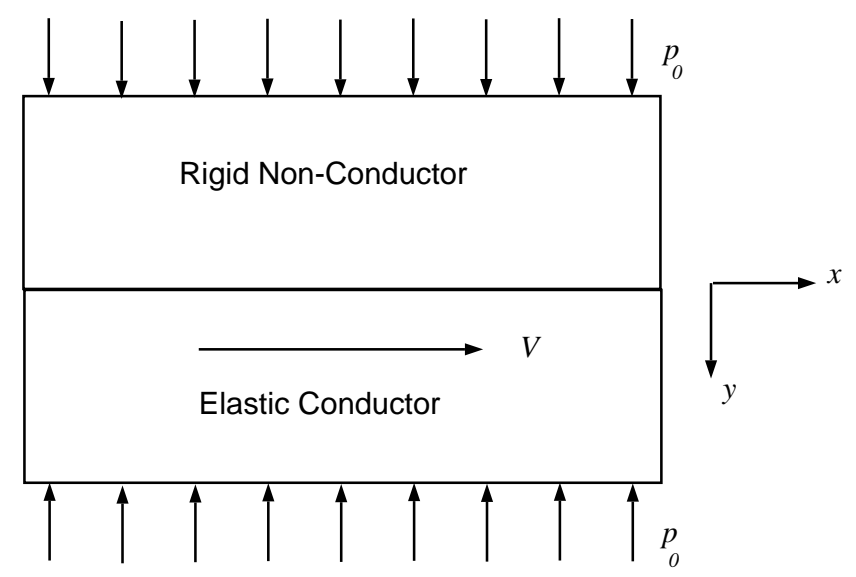

Fig. 1. Sliding contact of an elastic half-plane against a rigid plane surface.

and $k$ is the thermal diffusivity of the material. Notice that $\lambda$ is real for $b>-\mathrm{km}^{2}$, which includes all cases of unstable perturbations $(b>0)$. Eq. (16) has the two solutions $\theta=\exp ( \pm \lambda y)$, but we restrict attention to the negative exponent, since the perturbed temperature field is assumed to decay as $y \rightarrow \infty$. We therefore obtain

$$
T(x, y, t)=T_{0} \mathrm{e}^{-\lambda y+b t} \cos (m x),
$$

where $T_{0}$ is an arbitrary constant.

The temperature field (18) will cause thermal strains and a perturbation in contact pressure $p(x)$ will be needed to maintain contact with the rigid plane. This can be calculated using Eqs. (7), (8) of [11] as

$$
\begin{aligned}
p(x, t) & =\frac{2 \alpha(1+v) \mu m \mathrm{e}^{b t} \cos (m x)}{(1-v)} \int_{0}^{\infty} \exp (-m s) \theta(s) \mathrm{d} s \\
& =\frac{2 \alpha(1+v) \mu m T_{0} \mathrm{e}^{b t} \cos (m x)}{(1-v)(\lambda+m)},
\end{aligned}
$$

where $\alpha, \mu, v$ are the coefficient of thermal expansion, shear modulus and Poisson's ratio, respectively.

Substituting Eqs. (18), (19) into Eq. (13) and cancelling the common exponential and trigonometric factors, we obtain

$$
K \lambda=\frac{2 \alpha(1+v) \mu f V m}{(1-v)(\lambda+m)} .
$$

Solving for $\lambda$ and using Eq. (17), we finally obtain the exponential growth rate as

$$
b=k\left(\frac{2 \alpha(1+v) \mu f V m}{K(1-v)}-\frac{m^{2}}{2}-m \sqrt{\frac{m^{2}}{4}+\frac{2 \alpha(1+v) \mu f V m}{K(1-v)}}\right),
$$

where we have selected the root of Eq. (20) that corresponds to positive values of the decay constant $\lambda$. 


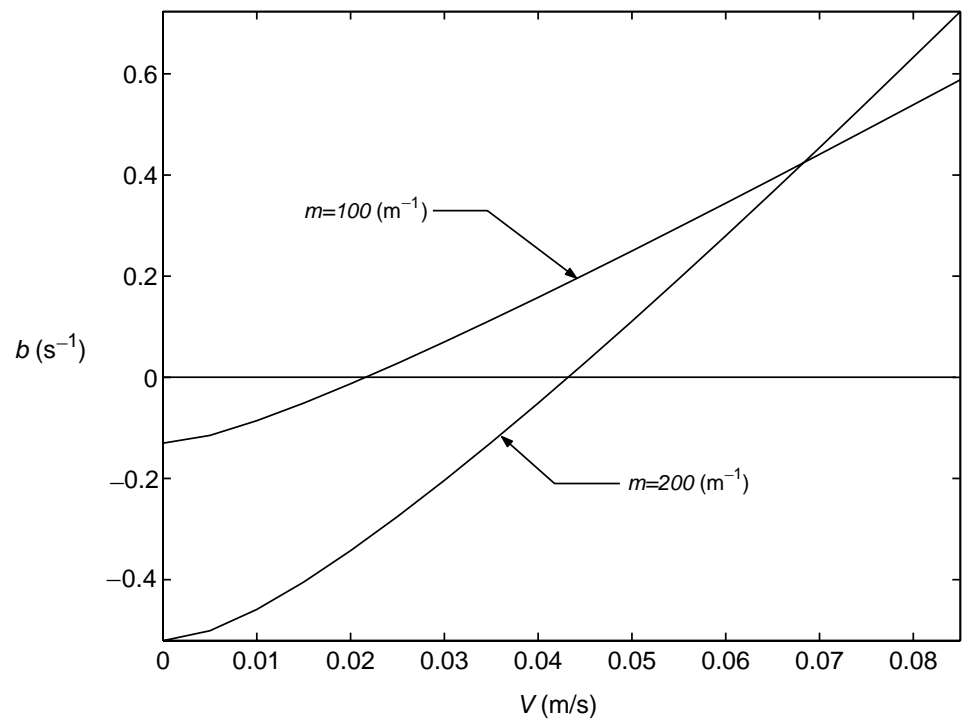

Fig. 2. Exponential growth rate as a function of sliding speed.

Fig. 2 shows the exponential growth rate $b$ as a function of speed for two different wavenumbers. The growth rate increases with speed as we should expect and is positive above a critical speed $V_{\text {cr }}$ given by

$$
V_{\mathrm{cr}}=\frac{K m(1-v)}{\alpha(1+v) \mu f} .
$$

The critical speed increases with wavenumber $m$ (long waves become unstable at lower speeds than short waves), but the growth rate curves intersect at higher speeds, showing that shorter wavelength perturbations may be expected to be more significant when the speed is significantly higher than critical.

Eq. (21) defines the growth rate $b(V)$ as a function of sliding speed $V$ and hence can be used in Eqs. (4), (5) to define an approximation to the evolution of a temperature or pressure perturbation of wavenumber $m$ when the speed varies with time.

\subsection{Direct finite element simulation}

To assess the accuracy of this approximation, a finite element solution was developed for the same transient thermoelastic contact problem using the commercial FE package ABAQUS. For sinusoidal initial temperature distributions, it is sufficient to model the finite region $0<x<l$ and use symmetric boundary conditions at the boundaries $x=0, l$, where $l=2 \pi / m$ is the wavelength of the perturbation. It is necessary to truncate the infinite dimension of the half-plane in the $y$-direction, but sinusoidal perturbations driven by surface effects tend to decay rapidly with depth, so if the region $0<y<2 l$ is discretized, the results are insensitive to the boundary condition imposed at $y=2 l$.

The finite element solution proceeds in two steps. At each time step, the instantaneous nodal temperatures are used in a thermomelastic contact solution to determine the contact pressure distribution. This distribution and the sliding speed are then assumed to remain constant during the ensuing time 


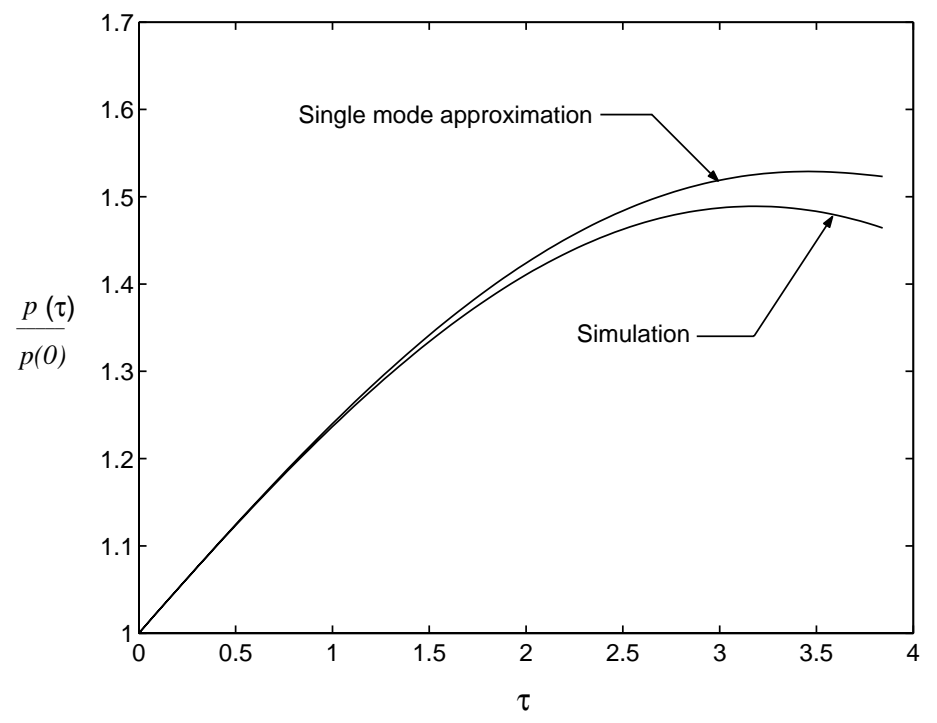

Fig. 3. Growth of the pressure perturbation with a linearly decreasing sliding speed. Comparison of numerical simulation and the one-term approximation of Eqs. (5), (21). The initial speed $V(0)=10 V_{\mathrm{cr}}$.

step, defining a heat conduction problem with a prescribed heat input at the sliding surface. The solution of this problem defines the nodal temperatures at the beginning of the next time step.

This algorithm was tested by comparison with the analytical solution of Section 3.1, which is exact if the sliding speed $V$ is constant and the initial temperature is taken to be

$$
T(x, y, t)=T_{0} \mathrm{e}^{-\lambda y} \cos (m x) .
$$

This test was used to select appropriate values of element size and time step to ensure accuracy and numerical stability in the simulation.

The one-term approximation (4), (5) was evaluated using an example in which the sliding speed decreases linearly with time from an initial value to zero, as in a typical brake or clutch application. The initial temperature perturbation was taken to have the form (23), with $\lambda$ corresponding to the initial speed $V(0)$. This example can be characterized in terms of the dimensionless initial speed $V(0) / V_{\text {cr }}$ and a dimensionless stopping time $\tau_{0}=\mathrm{km}^{2} t_{0}$, where $t_{0}$ is the actual stopping time.

Fig. 3 shows the amplitude of the pressure perturbation, normalized by the initial perturbation $p(0)$, as a function of dimensionless time $\tau=k m^{2} t$ for $V(0) / V_{\text {cr }}=10$ and $\tau_{0}=3.82$. The lower line corresponds to the finite element simulation and sufficient mesh and time step refinement were used for this to be regarded as the exact solution of the problem. The upper line is obtained from the approximate equation (5), using Eq. (21) for the growth rate $b(V)$.

The one-term approximation overestimates the growth of the perturbation, as suggested by the arguments of Section 2.1 , but only by about $14 \%$, which is quite good for such a simple approximation. A more extended series of comparisons showed that the stopping time $\tau_{0}$ has relatively little effect on the accuracy of the approximation, but the accuracy improves significantly for larger values of $V(0) / V_{\text {cr }}$, as shown for example in Fig. 4. This is to be anticipated, since the response is then dominated by the most rapidly growing eigenfunction that is modeled by the simple approximation. 


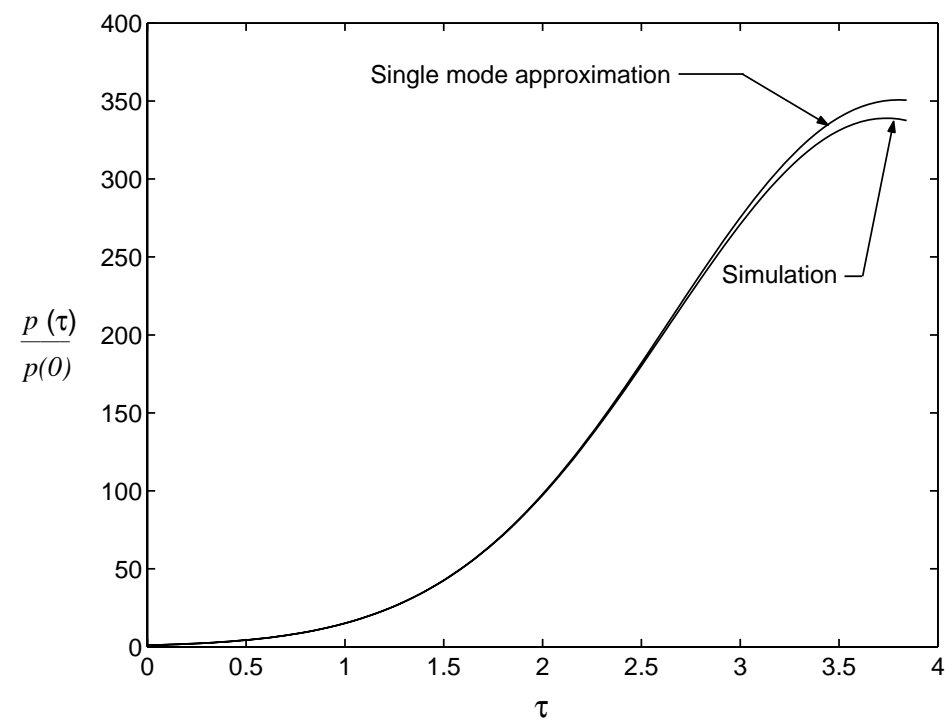

Fig. 4. Growth of the pressure perturbation for a larger initial speed $\left(V(0)=100 V_{\text {cr }}\right)$.

The dimensionless presentation permits the results of Figs. 3 and 4 to be applied to sinusoidal perturbations of any wavenumber and initial amplitude of contact pressure. However, the true evolution of the system depends on the exact initial distribution of temperature in the $y$-direction, rather than just on the initial contact pressure perturbation. This dependence on initial conditions was explored by using various perturbations of the form (23), with widely differing values of the spatial exponential decay rate $\lambda$. In all cases, the agreement with the one-term approximation was as good or better than that shown in Fig. 3, showing that the initial perturbation in pressure is the biggest determinant in the evolution of the system.

\section{Solution by discrete eigenfunction expansion}

If the geometry is discretized by the finite element method, the instantaneous temperature field can be characterized by a vector $\boldsymbol{\Theta}$ whose components are the $n$ nodal temperatures. We shall show in this section that the representation (1) then leads to an $n \times n$ eigenvalue problem for $\theta$ and it follows that there will be $n$ terms in the eigenfunction series (2). To develop the eigenvalue problem, we first approximate the temperature function $\theta(y)$ of Eq. (15) in the form

$$
\theta(y)=\sum_{i=1}^{n} N_{i}(y) \Theta_{i},
$$

where $\Theta_{i}$ are nodal temperatures and $N_{i}(y)$ are a set of $n$ shape functions. Applying the weighted residual method to Eq. (16), we obtain the set of equations

$$
\int W_{j}\left(\frac{\mathrm{d}^{2} \theta}{\mathrm{d} y^{2}}-\lambda^{2} \theta\right) \mathrm{d} y=0, \quad j=1, n,
$$


where $W_{j}$ is a set of linearly independent weighting functions. Integrating by parts yields

$$
-\frac{W_{j}(0) q_{0}}{K}-\int\left(\frac{\mathrm{d} W_{j}}{\mathrm{~d} y} \frac{\mathrm{d} \theta}{\mathrm{d} y}+\lambda^{2} W_{j} \theta\right) \mathrm{d} y=0,
$$

where

$$
q_{0}=-\left.K \frac{\mathrm{d} \theta}{\mathrm{d} y}\right|_{y=0}
$$

and we have assumed that there is no heat flux at the other (distant) boundary.

Substituting Eq. (24) into Eq. (26) and using the same functions $N_{i}$ as both shape and weighting functions, we obtain the matrix equation

$$
\left(\boldsymbol{M}+\lambda^{2} \boldsymbol{H}\right) \boldsymbol{\Theta}=\frac{q_{0} \boldsymbol{A}}{K},
$$

where

$$
M_{j i}=\int \frac{\mathrm{d} N_{j}}{\mathrm{~d} y} \frac{\mathrm{d} N_{i}}{\mathrm{~d} y} \mathrm{~d} y, \quad H_{j i}=\int N_{j} N_{i} \mathrm{~d} y
$$

and

$$
A_{j}=N_{j}(0)=\delta_{1 j}
$$

\subsection{The thermoelastic problem}

The nodal heat flux $q_{0}$ is proportional to the corresponding nodal contact pressure through the discrete equivalent of Eq. (13). A second equation linking the contact pressure to the temperature distribution can be obtained from the finite element solution of the thermoelastic contact problem.

We define the displacement field in the form

$$
u_{x}(x, y, t)=u(y) \mathrm{e}^{b t} \sin (m x), \quad u_{y}(x, y, t)=v(y) \mathrm{e}^{b t} \cos (m x),
$$

where the functions $u, v$ are written in the discrete form

$$
u(y)=\sum_{i=1}^{n} N_{i}(y) U_{i}, \quad v(y)=\sum_{i=1}^{n} N_{i}(y) V_{i}
$$

and $U_{i}, V_{i}$ are the components of the nodal displacement vector $\boldsymbol{U}$. The potential energy for the repeated segment $0<x<2 \pi / m$ can then be written as

$$
\Pi=\frac{1}{2} \int_{0}^{\infty} \int_{0}^{2 \pi / m}\left(\boldsymbol{\varepsilon}^{\mathrm{T}} \boldsymbol{\sigma}-\boldsymbol{\varepsilon}_{0}^{\mathrm{T}} \boldsymbol{\sigma}\right) \mathrm{d} x \mathrm{~d} y-\int_{0}^{2 \pi / m} u_{y}(x, 0, t) p(x, t) \mathrm{d} x,
$$

where $\boldsymbol{\sigma}=\left\{\sigma_{x}, \sigma_{y}, \tau_{x y}\right\}$ is the stress vector, $\boldsymbol{\varepsilon}=\left\{\varepsilon_{x}, \varepsilon_{y}, \gamma_{x y}\right\}$ is the strain vector,

$$
\boldsymbol{\varepsilon}_{0}=\alpha(1+v) T(x, y, t)\{1,1,0\}
$$

is the thermal strain and we have assumed traction-free conditions at the distant boundary. The stress and strain are related by $\boldsymbol{\sigma}=\boldsymbol{D}\left(\boldsymbol{\varepsilon}-\boldsymbol{\varepsilon}_{0}\right)$, where

$$
\boldsymbol{D}=\frac{E}{(1+v)(1-2 v)}\left[\begin{array}{ccc}
1-v & v & 0 \\
v & 1-v & 0 \\
0 & 0 & (1-2 v) / 2
\end{array}\right]
$$


Substituting Eqs. (32), (31) into the strain-displacement relations and Eqs. (24), (15) into Eq. (34), we obtain the discrete form of the strains as

$$
\boldsymbol{\varepsilon}=\sum_{i=1}^{n} \boldsymbol{B}_{i} \boldsymbol{U}_{i}, \quad \boldsymbol{\varepsilon}_{0}=\sum_{i=1}^{n} N_{i} \Theta_{i}\{1,1,0\} \mathrm{e}^{b t} \cos (m x)
$$

where

$$
\boldsymbol{B}_{i}=\left[\begin{array}{cc}
m N_{i} \cos (m x) & 0 \\
0 & \frac{\partial N_{i}}{\partial y} \cos (m x) \\
\frac{\partial N_{i}}{\partial y} \sin (m x) & -m N_{i} \sin (m x)
\end{array}\right] \mathrm{e}^{b t} .
$$

We then substitute Eqs. (35)-(37) into Eq. (33) and perform the integrations. Minimizing the resulting expression with respect to the nodal displacements $\boldsymbol{U}$ then yields the system of equations

$$
\boldsymbol{K} U=\boldsymbol{G} \boldsymbol{\Theta}+p_{0} \boldsymbol{A},
$$

where $\boldsymbol{A}$ is defined in Eq. (30),

$$
\boldsymbol{K}=\int_{0}^{\infty} \tilde{\boldsymbol{B}}^{\mathrm{T}} \boldsymbol{D} \tilde{\boldsymbol{B}} \mathrm{d} y, \quad \boldsymbol{G}=\int_{0}^{\infty} \tilde{\boldsymbol{B}}^{\mathrm{T}} \boldsymbol{D} \tilde{\boldsymbol{C}} \mathrm{d} y
$$

and

$$
\tilde{\boldsymbol{B}}=\left[\begin{array}{llll}
\tilde{\boldsymbol{B}}_{1} & \tilde{\boldsymbol{B}}_{2} & \ldots & \tilde{\boldsymbol{B}}_{n}
\end{array}\right], \quad \tilde{\boldsymbol{C}}=\left[\begin{array}{llll}
\tilde{\boldsymbol{C}}_{1} & \tilde{\boldsymbol{C}}_{2} & \ldots & \tilde{\boldsymbol{C}}_{n}
\end{array}\right]
$$

with

$$
\tilde{\boldsymbol{B}}_{i}=\left[\begin{array}{cc}
m N_{i} & 0 \\
0 & \frac{\partial N_{i}}{\partial y} \\
\frac{\partial N_{i}}{\partial y} & -m N_{i}
\end{array}\right], \quad \tilde{\boldsymbol{C}}_{i}=N_{i}\left\{\begin{array}{l}
1 \\
1 \\
0
\end{array}\right\} .
$$

The half-plane is in contact with a rigid plane at $y=0$ and hence $U_{1}=0$ in Eq. (32). The remaining $2 n-1$ unknown nodal displacements can be eliminated from the $2 n$ linear algebraic equations (38) to yield a single equation for $p_{0}$ in terms of $\boldsymbol{\Theta}$ which can be written in the symbolic form

$$
p_{0}=\boldsymbol{L} \boldsymbol{\Theta} .
$$

The frictional heating relation $q_{0}=f V p_{0}$ and Eqs. (28), (42), (17) can then be used to eliminate $p_{0}, q_{0}, \lambda$, leading to the generalized $n \times n$ linear eigenvalue equation

$$
\left(\frac{f V}{K} \boldsymbol{A} L-\boldsymbol{M}-m^{2} \boldsymbol{H}\right) \boldsymbol{\Theta}=\frac{b}{k} \boldsymbol{H} \boldsymbol{\Theta} .
$$

This equation will have $n$ eigenvalues and eigenfunctions, which we denote by $b_{k}, \hat{\Theta}_{i}^{k}$, respectively. A general solution for the evolution of the nodal temperatures $\Theta_{i}(t)$ at constant speed can then be 
written as the eigenfunction series

$$
\Theta_{i}(t)=\sum_{k=1}^{n} C_{k} \hat{\Theta}_{i}^{k} \mathrm{e}^{b_{k} t},
$$

where the $n$ constants $C_{k}$ are to be determined from the $n$ equations defining the initial nodal temperatures $\Theta_{i}(0)$. This representation can be generalized to cases where the speed is piecewise constant, as in Section 2.1 above. Also a reduced order model can be constructed by arranging the eigenvalues in order of decreasing real part and truncating the series in Eq. (44) at some value less than $n$.

\section{Results}

The eigenfunction expansion (2) provides an exact solution to the transient problem as long as the sliding speed is constant and all $n$ eigenfunctions are included. For cases involving variable speed $V(t)$, the approximation inherent in the piecewise constant representation of Eqs. (6) $-(8)$ was assessed by comparing the predictions of the eigenfunction expansion with the direct numerical simulation of Section 3.2.

Fig. 5 shows the predicted maximum surface temperatures for uniform deceleration with dimensionless initial speed $V(0) / V_{\text {cr }}=100$ and dimensionless stopping time $\tau_{0}=3.82$. Extremely good agreement $( \pm 1 \%)$ was obtained by dividing the stop into as few as 20 equal time steps. With 10 time steps, the maximum error increased to $( \pm 4 \%)$, but even this would be acceptable in many engineering applications. Results for the maximum contact pressure were even better, with only $1 \%$ error in the prediction with as few as 5 time steps.

A parametric study of other cases showed that the number of time steps required for a given level of accuracy is quite insensitive to the parameters $V(0) / V_{\text {cr }}$ and $\tau_{0}$. This is perhaps to be expected, since the source of error is the change in speed during the time step and the use of a constant number of steps during the stop preserves the same proportional change in speed during corresponding steps. In all cases, 20 equal time steps during the stop were sufficient to give excellent accuracy.

\subsection{The reduced order model}

The eigenfunction expansion can be truncated at any number of terms to create a reduced order model of the system. For this purpose, the eigenfunction series must be ranked in order to determine which terms should be retained. An obvious choice is to rank the functions in order of decreasing exponential growth rate $b_{i}$. This works well during the growth phase, when the sliding speed is above the critical speed, but when the speed falls below critical the dominant eigenvalues tend to cluster together. In this range, better results were obtained by retaining those terms with the larger multiplying coefficients $\left(C_{k}\right.$ in Eq. (44)) after the eigenfunctions have been normalized.

Fig. 6 shows the maximum surface temperature predicted by the reduced order model for various numbers of terms in the truncated series. A single term gives quite good results during the growth phase, but is unable to describe the later part of the stop, principally because of the clustering of eigenvalues described above. Quite good accuracy is obtained using a 20 term series. By comparison, 


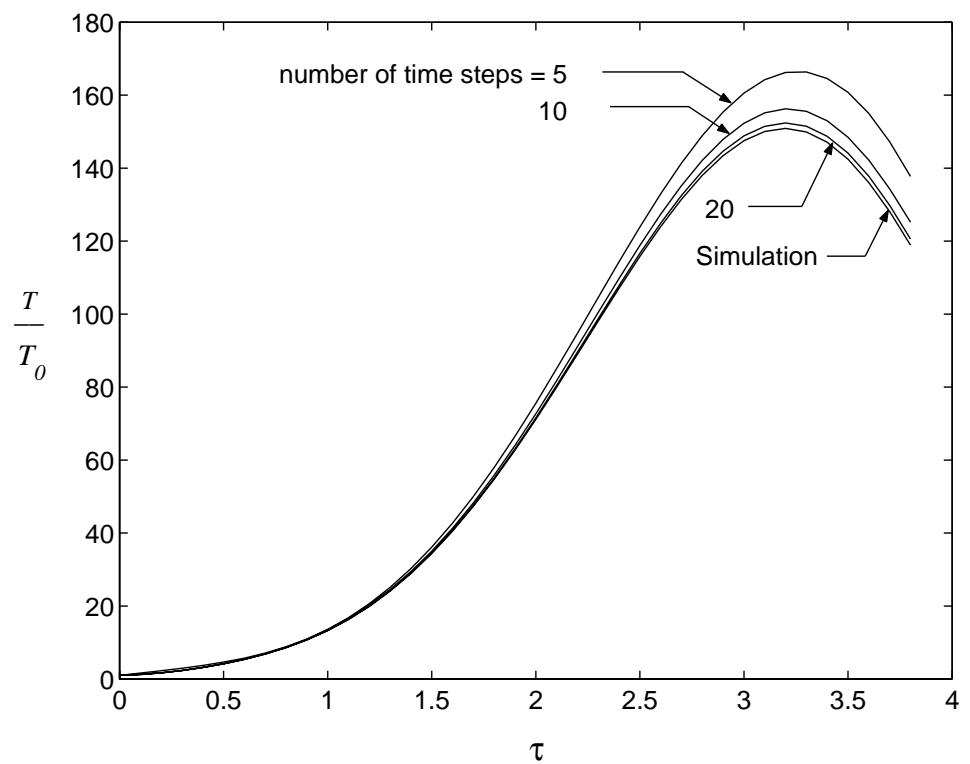

Fig. 5. Effect of time step on the accuracy of the eigenfunction expansion $\left(V(0) / V_{\text {cr }}=100\right)$.

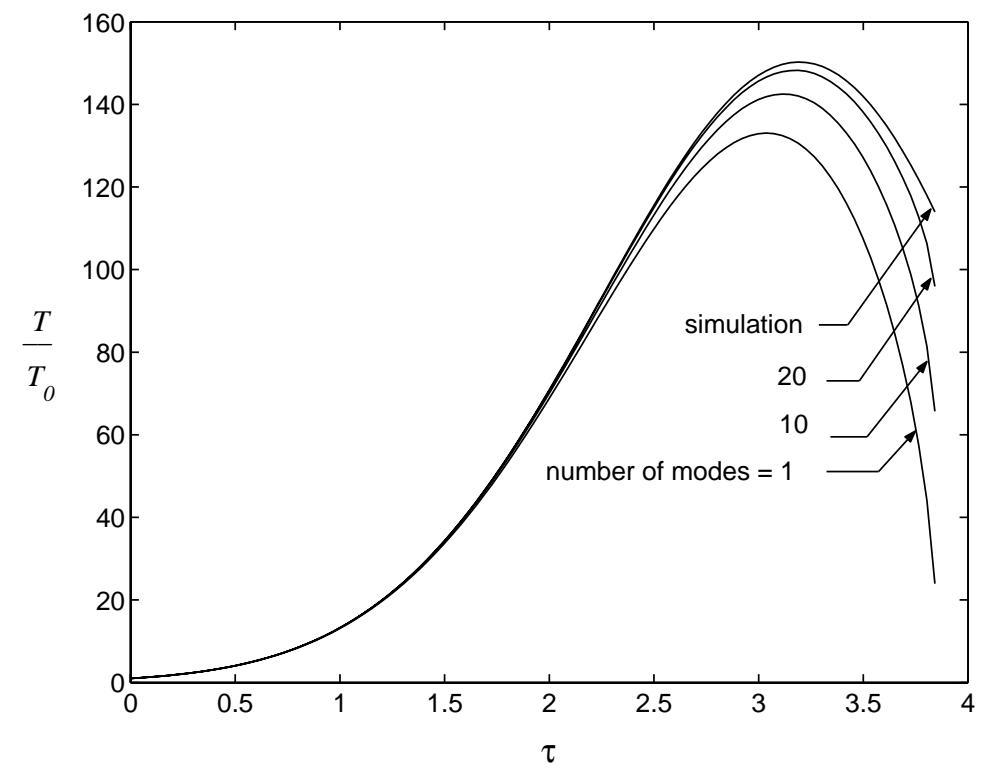

Fig. 6. Effect of the number of terms in the reduced order model on the accuracy of the eigenfunction expansion $\left(V(0) / V_{\text {cr }}=100\right)$. 
the exact solution in this case involves 200 terms, corresponding to the 200 nodes used in the $y$-direction discretization of the temperature field.

\section{Conclusions}

In this paper, we have explored several approximate solutions to the transient thermoelastic contact problem with frictional heating, based on the concept of an eigenfunction expansion. If the system is discretized using the finite element method, a complete eigenfunction expansion provides an excellent computationally efficient solution with quite coarse discretization in the time domain.

Truncated series (reduced order models) provide excellent approximations during the first part of a brake or clutch engagement, during which the sliding speed is above the critical speed. In fact, a single term approximation works quite well in this range and this method can be used to obtain an integral expression for the evolution of the perturbation in temperature or pressure. Towards the end of the engagement when the speed is sub-critical, the eigenvalues become clustered and substantially more terms are needed to obtain accurate solutions.

\section{Acknowledgements}

The authors are pleased to acknowledge support from the Raytech Composites Inc. and from the National Science Foundation under contract number CMS-9619527.

\section{References}

[1] Barber JR. Thermoelastic instabilities in the sliding of conforming solids. Proceedings of the Royal Society of London, Series A 1969;312:381-94.

[2] Lee K, Dinwiddie RB. Conditions of frictional contact in disk brakes and their effects on brake judder. SAE 980598, 1998.

[3] Kao TK, Richmond JW, Douarre A. Brake disc hot spotting and thermal judder: an experimental and finite element study. International Journal of Vehicle Design 2000;23:276-96.

[4] Kubota M, Suenaga T, Doi K. A study of the mechanism causing high speed brake judder. SAE 980594, 1998.

[5] Burton RA, Nerlikar V, Kilaparti SR. Thermoelastic instability in a seal-like configuration. Wear 1973;24:177-88.

[6] Du S, Zagrodzki P, Barber JR, Hulbert GM. Finite element analysis of frictionally-excited thermoelastic instability. Journal of Thermal Stresses 1997;20:185-201.

[7] Yi Y-B, Barber JR, Zagrodzki P. Eigenvalue solution of thermoelastic instability problems using Fourier reduction. Proceedings of the Royal Society of London, Series A 2000;456:2799-821.

[8] Barber JR, Beamond TW, Waring JR, Pritchard C. Implications of thermoelastic instability for the design of brakes. ASME Journal of Tribology 1985;107:206-10.

[9] Zagrodzki P. Analysis of thermomechanical phenomena in multidisc clutches and brakes. Wear 1990;140:291-308.

[10] Johansson L. Model and numerical algorithm for sliding contact between two elastic half-planes with frictional heat generation and wear. Wear 1993;160:77-93.

[11] Barber JR, Hector LG. Thermoelastic contact problems for the layer. ASME Journal of Applied Mechanics 1999;66:806-9. 